\title{
A Patient with a Ruptured Blood Report Blister-like Aneurysm of the Internal Carotid Artery in Whom Two LVIS Stents Were Inserted
}

Ryotaro Suzuki, Tomoji Takigawa, Toru Anazawa, Nobuo Shirasaka, Yoshiyuki Matsumoto, Yoshiko Fujii, Yasuhiko Nariai, Yoshiki Sugiura, Yosuke Kawamura, Issei Takano, Yoshihiro Tanaka, Masaya Nagaishi, Akio Hyodo, and Kensuke Suzuki

Objective: We report a case in which multiple overlapping low-profile visualized intraluminal support (LVIS) stents were used as monotherapy for ruptured blood blister-like aneurysm (BBA) of the internal carotid artery (ICA).

Case Presentation: A 48-year-old female presented to the emergency room with acute-onset headache. She was alert without neurological deficit with subarachnoid hemorrhage (SAH) Hunt and Kosnik grade I. Emergency angiography revealed a BBA from the supraclinoid ICA. This aneurysm had a small diameter, which makes coil embolization difficult. Therefore, we planned to use multiple overlapping LVIS stents as monotherapy for the ruptured aneurysm. The postoperative course was uneventful without rebleeding. The patient exhibited no neurological deficits on the clinical follow-up at 1 year. Conclusion: Multiple overlapping LVIS stents as monotherapy is useful for ruptured BBAs of the ICA.

Keywords > subarachnoid hemorrhage, ruptured aneurysm, blood blister-like aneurysm, multiple overlapping stent, low-profile visualized intraluminal support stents

\section{Introduction}

Blood blister-like aneurysms (BBAs) account for $0.5 \%-2 \%$ of all ruptured aneurysms. A previous study reported that the BBA-related mortality rate was particularly high. ${ }^{1)}$ BBAs are small aneurysms that develop at bifurcation-free sites such as the middle cerebral artery and anterior communicating artery. The most frequent site is the supraclinoid portion of the internal carotid artery (ICA)., ${ }^{2,3)}$ BBAs are characterized by a hemispherical shape, wide neck, difficulty in identifying the neck from a parent artery, shape changes in a short period, and fragility-related risk of rebleeding or

$\overline{\text { Department of Neurosurgery, Dokkyo Medical University Saitama }}$ Medical Center, Koshigaya, Saitama, Japan

Received: July 25, 2019; Accepted: December 24, 2019 Corresponding author: Ryotaro Suzuki. Department of Neurosurgery, Dokkyo Medical University Saitama Medical Center, 2-1-50 Minamikoshigaya, Koshigaya, Saitama 343-0855, Japan Email: ryo096943@gmail.com

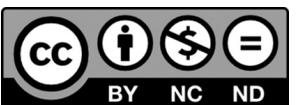

This work is licensed under a Creative Commons Attribution-NonCommercialNoDerivatives International License.

C2020 The Japanese Society for Neuroendovascular Therapy enlargement, ${ }^{4)}$ differing from saccular aneurysms, which are routinely observed.

In patients with BBAs, rebleeding in the early stage is common. To prevent rebleeding, BBAs should be treated as early as possible. ${ }^{5)}$ Treatment methods include craniotomy, such as direct clipping, suturing, clipping after wrapping, and encircling clipping, and endovascular treatment, such as endovascular or surgical trapping with/without bypass, coil embolization, stenting, that with a flow-diverting stent, and that with multiple overlapping stents. However, there is no consensus regarding optimal treatment. ${ }^{\text {) }}$

We report a patient in whom a ruptured BBA of the ICA was treated using two low-profile visualized intraluminal support (LVIS) stents (Microvention-Terumo, Tustin, CA, USA) without combining them with a coil.

\section{Case Presentation}

The patient was a 48-year-old female. She suddenly developed headache, and was brought to our hospital by ambulance. Her medical history was not contributory. Cephalic computed tomography (CT) did not reveal subarachnoid hemorrhage (SAH). Cephalic magnetic resonance imaging (MRI)-fluid-attenuated inversion recovery (FLAIR) 
A

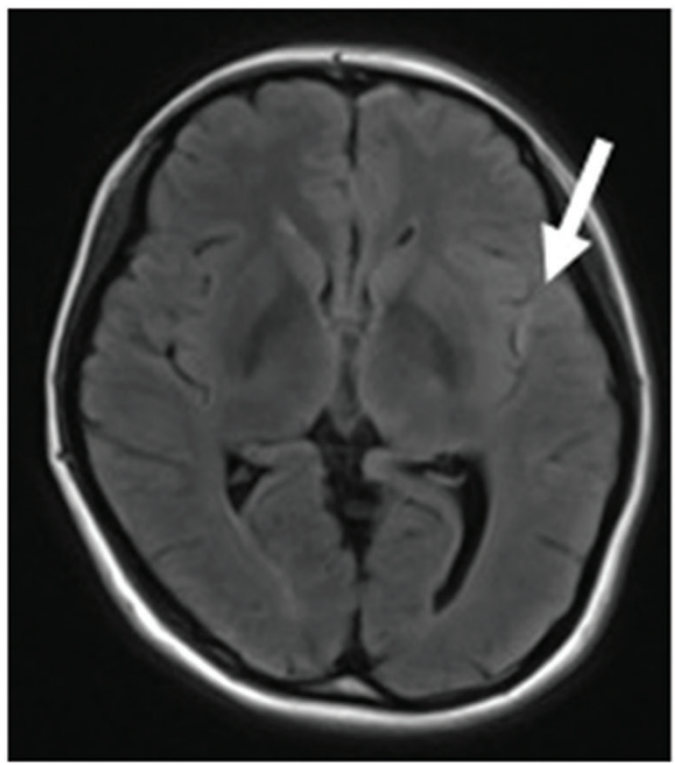

B

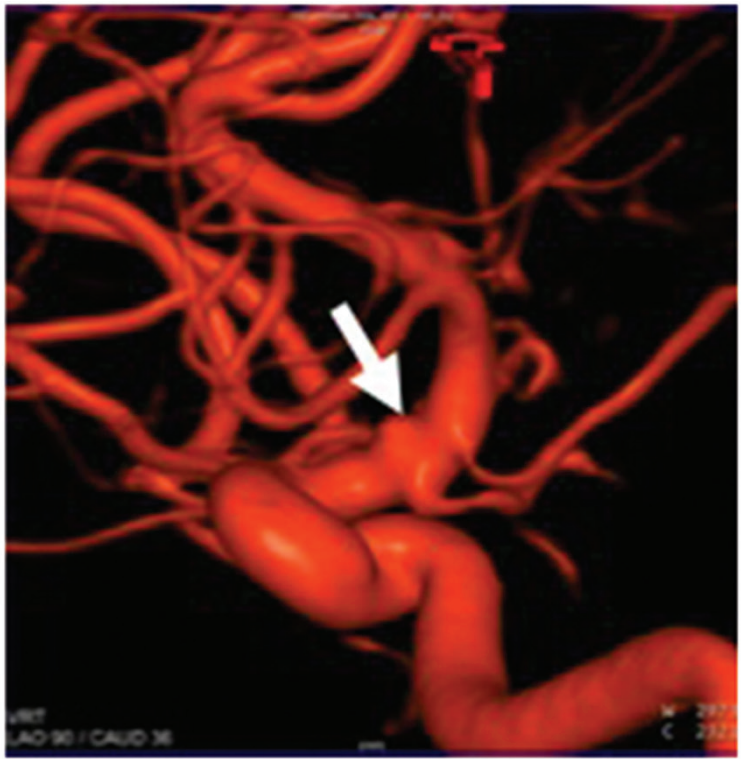

Fig. 1 (A) MRI-fluid-attenuated inversion recovery (FLAIR) on admission showed subarachnoid hemorrhage at the left Sylvian fissure. (B) Angiography. Left internal carotid angiography (3D reconstruction image) revealed a blood blister-like aneurysm in the supraclinoid portion of the left internal carotid artery (white arrow).

demonstrated SAH at the left Sylvian fissure (Fig. 1A). Consciousness was clear, and there was no symptom other than headache. There were no neurological abnormalities. A diagnosis of Hunt and Kosnik grade 1, World Federation of Neurosurgical Societies (WFNS) grade $1 \mathrm{SAH}$ was made. On magnetic resonance angiography (MRA), the source of hemorrhage was unclear. Angiography revealed aneurysm of the supraclinoid portion of the ICA, with a maximum diameter of $2.4 \mathrm{~mm}$ (Fig. 1B). There was no other aneurysm, suggesting rupture at this site. However, SAH was mild (Hunt and Kosnik grade 1), and we selected surgical intervention through angiography after 1 week, considering the risks of all acute-phase treatments for the following reasons: the therapeutic risk of ICA trapping combined with high-flow bypass under craniotomy is high; ICA trapping as endovascular treatment may cause cerebral infarction because the collateral pathway cannot be evaluated; and coil embolization was impossible due to a small aneurysm. Sedation and hypotensive treatment were continued, and there was no rebleeding. Angiography 7 days after admission did not demonstrate any changes in the aneurysmal size or shape. Although hemorrhage was slight, treatment for the prevention of rebleeding was selected for the following reasons: there was no source of hemorrhage other than the hemorrhagic site, and rebleeding in the presence of a BBA may lead to a fatal course. Due to the small, wide-neck aneurysm, a coil-free procedure with two LVIS stents was adopted to obtain flow diverter effects.

To prevent ischemic complications, $100 \mathrm{mg}$ of aspirin was administered immediately before surgery, and loading with $300 \mathrm{mg}$ of clopidogrel was performed. Therapy with $100 \mathrm{mg} /$ day of aspirin and $75 \mathrm{mg} /$ day of clopidogrel was continued from the following day. Treatment was performed 8 days after admission. Under general anesthesia, a 6-Fr shuttle sheath (Cook Medical, Indianapolis, IN, USA) was inserted into the left common carotid artery after heparinization, and a 6-Fr FUBUKI (Asahi Intecc, Aichi, Japan) was inserted into the left ICA. A Headway 21 (MicroventionTerumo, Tustin, CA, USA) was guided to the middle cerebral artery distal to the aneurysm using a CHIKAI 14 (Asahi Intecc). An LVIS blue $3.5 \mathrm{~mm} \times 12 \mathrm{~mm}$ was deployed/inserted to cover the entire aneurysm. In addition, an LVIS blue $3.5 \mathrm{~mm} \times 17 \mathrm{~mm}$ (second stent) was similarly deployed/inserted to cover the entire first stent. There were no changes in the visualization of the aneurysm on angiography immediately after insertion (Fig. 2A).

After surgery, there was no new appearance of neurological abnormalities. Postoperative diffusion-weighted MRI did not reveal acute cerebral infarction (Fig. 3). On MRA, there was no problem other than stent artifacts, and the visualization of peripheral arteries was favorable. On angiography 14 days after surgery, the visualization 
A

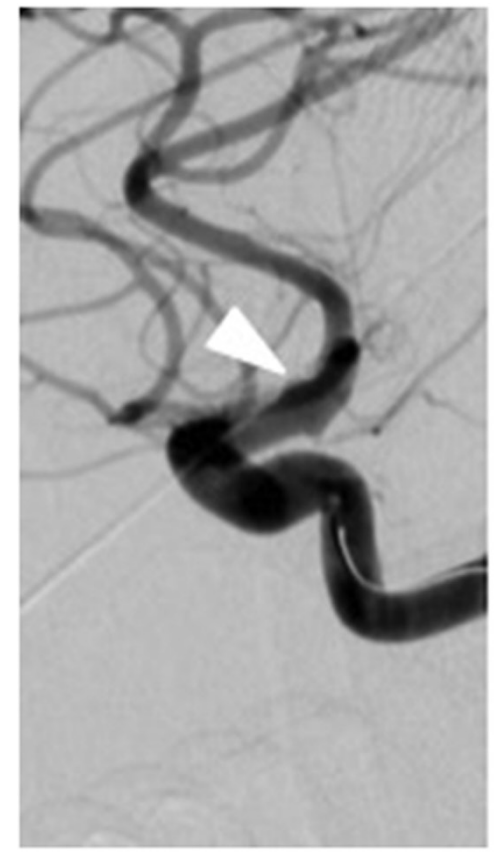

$\mathrm{B}$

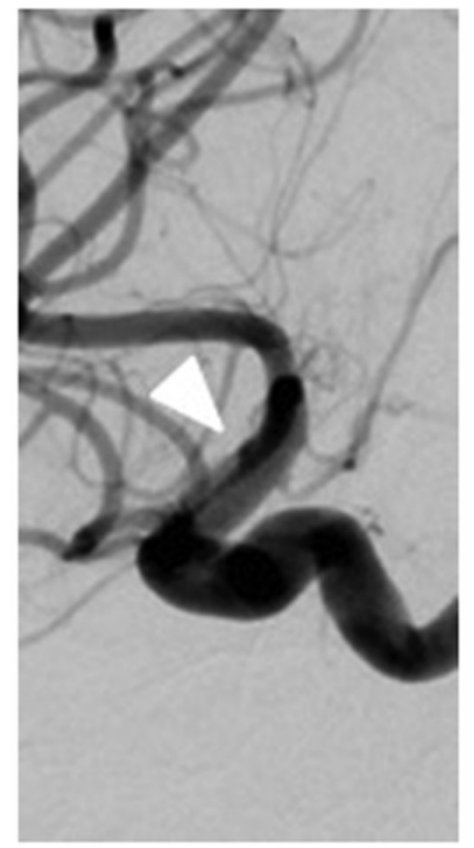

C

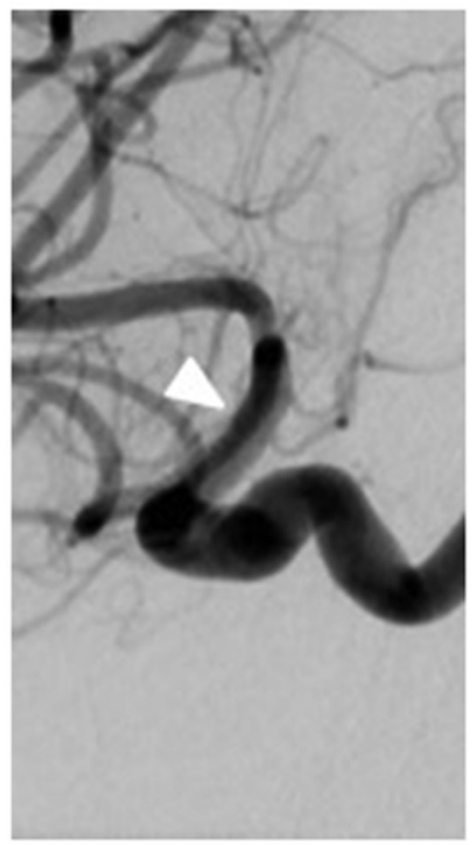

Fig. 2 (A) Angiography immediately after surgery. Left internal carotid angiography showed that the aneurysm remained (white arrow head). (B) Angiography 14 days after surgery. On left internal carotid angiography, visualization of the aneurysm was slightly attenuated and slight intra-aneurysmal retention of contrast medium was noted (white arrow head). (C) Angiography 4 months after surgery. Left internal carotid angiography confirmed the disappearance of the aneurysm and absence of intra-stent stenosis (white arrow head).

of the aneurysm was slightly attenuated, and slight intra-aneurysmal retention of contrast medium was noted (Fig. 2B). There was no vasospasm-related symptom or hydrocephalus. The course was favorable. The patient was discharged 37 days after admission with no new neurological abnormalities.

Angiography was performed 4 months after surgery to confirm the disappearance of the aneurysm. Furthermore, the absence of intra-stent stenosis was confirmed (Fig. 2C). The administration of clopidogrel, as an antiplatelet drug, was completed 6 months after surgery. Subsequently, aspirin alone was continuously administered for 1 year. There has been no rebleeding or new neurological abnormalities during the 1-year follow-up after treatment; the outcome has been favorable.

\section{Discussion}

Treatment of ruptured BBAs of the ICA is challenging. $\mathrm{Ji}$ et al. classified treatment methods into three types ${ }^{6}$ : surgical BBA occlusion with ICA preservation (direct clipping, suturing, clipping after wrapping, or clipping after suturing), BBA occlusion by endovascular treatment with ICA preservation (coil embolization, stenting, flow-diverting

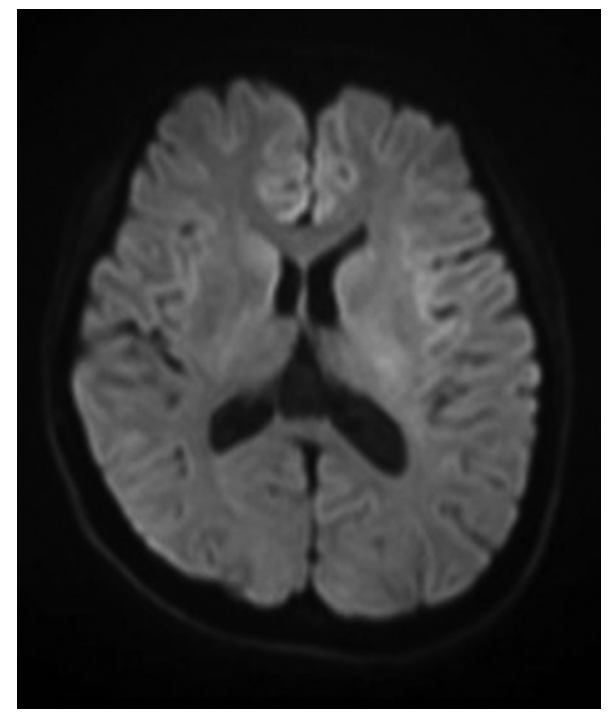

Fig. 3 Postoperative diffusion-weighted MRI did not reveal acute-phase cerebral infarction.

stent insertion, or multiple overlapping stent insertion), and surgical or endovascular trapping of a parent artery combined with bypass. The incidence of complications is high regardless of the type of treatment, and no treatment method has been established. 
Table 1 Review of multiple overlapping Stent as monotherapy for ruptured BBA of the ICA

\begin{tabular}{|c|c|c|c|c|c|c|c|c|}
\hline Author & Age & Gender & $\begin{array}{l}\text { Hunt and } \\
\text { Kosnik } \\
\text { grade }\end{array}$ & Stent & $\begin{array}{l}\text { Angiographic } \\
\text { follow-up } \\
\text { (month) }\end{array}$ & $\begin{array}{l}\text { Aneurysm } \\
\text { occlusion }\end{array}$ & $\begin{array}{l}\text { Outcome } \\
\text { (mRS) }\end{array}$ & \\
\hline Fiorella $\mathrm{D}$ et al. & 61 & $\mathrm{~F}$ & NA & $\mathrm{N} \times 3$ & 9 & + & 0 & \\
\hline Neurosurgery & 51 & $\mathrm{~F}$ & NA & $\mathrm{N} \times 2$ & 14 & + & 0 & \\
\hline $2006^{16)}$ & 46 & M & NA & $\mathrm{N} \times 2$ & 4 & + & 0 & \\
\hline Gaughen et al. & 66 & $\mathrm{~F}$ & I & $E \times 2$ & 7 & + & 1 & \\
\hline Am J Neuroradiol & 36 & $\mathrm{~F}$ & III & $E \times 2$ & 4 & + & 1 & \\
\hline \multirow[t]{3}{*}{$2010^{17)}$} & 47 & $\mathrm{~F}$ & II & $E \times 2$ & 10 & + & 1 & \\
\hline & 40 & M & II & $\mathrm{N} \times 2$ & 12 & + & 1 & \\
\hline & 42 & M & III & $E \times 2$ & 1 & - & 3 & Rebleeding \\
\hline Meckel et al. Am J & 41 & M & IV & $\mathrm{N} \times 2$ & 1 & - & 2 & Regrowth \\
\hline Neuroradiol 2011'18) & 52 & $\mathrm{~F}$ & I & $W \times 2$ & 1 & - & 2 & Regrowth \\
\hline Walsh KM et al. & 60 & $\mathrm{~F}$ & NA & $\mathrm{N} \times 2$ & 10 & + & 1 & \\
\hline $\mathrm{J}$ Neurointervent & 80 & $\mathrm{~F}$ & II & $N+E$ & 6 & + & 1 & \\
\hline \multirow[t]{5}{*}{ Surg 201419) } & 55 & $\mathrm{~F}$ & III & $N+E$ & 1 & - & 2 & Regrowth \\
\hline & 52 & M & III & $E \times 2$ & 12 & + & 1 & \\
\hline & 53 & M & II & $E \times 3$ & 3 & + & 1 & \\
\hline & 60 & $\mathrm{~F}$ & II & $E \times 2$ & 24 & + & 1 & \\
\hline & 71 & M & III & $E \times 2$ & 12 & + & 2 & \\
\hline Present case & 45 & $\mathrm{~F}$ & I & $\mathrm{L} \times 2$ & 4 & + & 0 & \\
\hline
\end{tabular}

As endovascular treatment, coil embolization, stenting, flow-diverting stent insertion, or multiple overlapping stent insertion is performed. Regarding coil embolization, some studies reported that this procedure was useful for treating large, saccular aneurysms, ${ }^{7,8)}$ but there are few patients with small, wide-neck BBAs for whom this procedure is indicated. ${ }^{1)}$

Matsubara et al. ${ }^{7)}$ reported that coil embolization was possible in 17 of 22 patients with BBAs. According to Lim et al., ${ }^{8)}$ it was possible in 30 of 33 patients with BBAs. Furthermore, these studies indicated the usefulness of stentassisted coil embolization. Another study reported the usefulness of coil embolization for the treatment of acutephase ruptured BBAs. ${ }^{9)}$ However, the aneurysmal size may rapidly increase after stent-assisted coil embolization or rebleeding may occur in the early phase. Moreover, several studies reported a high recurrence rate. ${ }^{10,11)}$ In the present case, coil embolization alone was considered to be impossible due to the small ( $\leq 3 \mathrm{~mm})$, wide-neck aneurysm.

Flow-diverting stents facilitate parent vessel wall endothelial formation, providing an ideal treatment method for ruptured BBAs. ${ }^{12)}$ Several studies reported a high aneurysmal occlusion rate and long-term favorable outcome after flow diverter treatment for ruptured BBAs. ${ }^{13-15)}$ Although its usefulness was suggested, flow diverter treatment for ruptured or small aneurysms is not covered by health insurance in Japan, and neither are assist stents. However, an assist stent was used based on approval by the ethics review board of our hospital due to the necessity of treatment.

As alternative treatment in cases in which a flow diverter cannot be used, several studies examined the usefulness of coil-free treatment with multiple overlapping stents alone for ruptured BBAs. ${ }^{16-19)}$ The background of the 18 patients, including our patient, is presented in Table 1. Rebleeding was noted in one patient and aneurysmal enlargement in three, with a mean follow-up of 7.9 months. In the other patients, favorable aneurysmal occlusion was achieved. In most patients, Enterprise (Johnson \& Johnson Codman, Miami, FL, USA) or Neuroform (Boston Scientific/Target Therapeutics, Fremont, CA, USA) stents were used. An LVIS stent was used only in the present case. It was a nitinol, self-expandable, closed-cell-structure blade stent, with a metal coverage of approximately $23 \%$. This percentage was higher than those of an Enterprise (10\%) or Neuroform (11\%), but it was lower than that of a Pipeline embolization device (PED; Medtronic Neurovascular, Irvine, CA, USA) (approximately 30\%-35\%). The number of stents to be used remains to be clarified. Wang et al. performed computational fluid dynamics (CFD) analysis, and compared the flow diverter effects of an LVIS, Enterprise, and Pipeline. ${ }^{20)}$ As CFD parameters, they measured the velocity, wall share stress (WSS), and pressure, and found that double LVIS stents significantly reduced the WSS and velocity in comparison with a single Pipeline. As a result, the flow 
diverter effects of double LVIS stents were similar to those of a Pipeline.

The limitations of multiple overlapping stent treatment include a long interval until the disappearance of the aneurysm, during which rebleeding or enlargement may occur. To prevent ischemic complications during/after multiple overlapping stent treatment, the combination of two antiplatelet drugs is selected in accordance with treatment with a flow diverter; therefore, hemorrhagic complications may develop. According to previous studies, a mean interval of 8.8 months was required until the disappearance of the aneurysm. In the present case, approximately 4 months were required until its disappearance. During this period, rebleeding is possible and careful follow-up is necessary. In the present case, careful follow-up by resting and hypotensive therapy was conducted in the acute phase, and strict hypotensive therapy was also continued after this phase. We planned to perform ICA trapping combined with high-flow bypass under craniotomy or ICA trapping by endovascular treatment at the time of rebleeding. Furthermore, enlargement of an aneurysm after insertion is difficult to treat, and coil embolization, as an additional option, or additional stenting must be selected; the number of treatment options is limited. We planned to perform additional stent insertion for an aneurysm remaining in the chronic phase. Combination therapy with two antiplatelet drugs may induce hemorrhagic complications; therefore, it should be switched to monotherapy if possible. However, a consensus regarding the period of combination therapy with two antiplatelet drugs has not been reached. In the present case, this combination therapy was switched to monotherapy 6 months after its start, and there have been no ischemic or hemorrhagic complications.

\section{Conclusion}

We reported a case of coil-free stenting for a ruptured BBA of the ICA. In patients with ruptured or small aneurysms, coil embolization is difficult, and the insertion of several LVIS stents in the subacute phase may be useful. However, this treatment has not been established, and ischemic complications or hemorrhage related to the combination of two antiplatelet drugs may develop. In the future, its efficacy and safety should be further investigated.

\section{Disclosure Statement}

We declare no conflict of interest.

\section{References}

1) Meling TR, Sorteberg A, Bakke SJ, et al: Blood blister-like aneurysms of the internal carotid artery trunk causing subarachnoid hemorrhage: treatment and outcome. J Neurosurg 2008; 108: 662-671.

2) Szmuda T, Sloniewski P, Waszak PM, et al: Towards a new treatment paradigm for ruptured blood blister-like aneurysms of the internal carotid artery? A rapid systematic review. J Neurointerv Surg 2016; 8: 488-494.

3) Peschillo S, Cannizzaro D, Caporlingua A, et al: A systematic review and meta-analysis of treatment and outcome of blister-like aneurysms. AJNR Am J Neuroradiol 2016; 37: 856-861.

4) Horie N, Morikawa M, Fukuda S, et al: Detection of blood blister-like aneurysm and intramural hematoma with highresolution magnetic resonance imaging. J Neurosurg 2011; 115: 1206-1209.

5) Le Feuvre DE, Taylor AG: The management of very small/ blister internal carotid artery aneurysms. Interv Neuroradiol 2011; 17: 431-434.

6) Ji T, Guo Y, Huang X, et al: Current status of the treatment of blood blister-like aneurysms of the supraclinoid internal carotid artery: a review. Int J Med Sci 2017; 14: 390-402.

7) Matsubara N, Miyachi S, Tsukamoto N, et al: Endovascular coil embolization for saccular-shaped blood blister-like aneurysms of the internal carotid artery. Acta Neurochir (Wien) 2011; 153: 287-294.

8) Lim YC, Kim BM, Suh SH, et al: Reconstructive treatment of ruptured blood blister-like aneurysms with stent and coil. Neurosurgery 2013; 73: 480-488.

9) Korja M, Rautio R, Valtonen S, et al: Primary treatment of ruptured blood blister-like aneurysms with stent-assisted coil embolization: report of two cases. Acta Radiol 2008; 49: 180-183.

10) Ahn JY, Cho JH, Jung JY, et al: Blister-like aneurysms of the supraclinoid internal carotid artery: challenging endovascular treatment with stent-assisted coiling. J Clin Neurosci 2008; 15: 1058-1061.

11) Ihn YK, Kim SH, Sung JH, et al: The efficacy of endovascular treatment of ruptured blood blister-like aneurysms using stent-assisted coil embolization. Interv Neuroradiol 2012; 18: 432-441.

12) Heiferman DM, Billingsley JT, Kasliwal MK, et al: Use of flow-diverting stents as salvage treatment following failed stent-assisted embolization of intracranial aneurysms. $\mathrm{J} \mathrm{Neu}$ rointerv Surg 2016; 8: 692-695.

13) Çinar C, Oran I, Bozkaya H, et al: Endovascular treatment of ruptured blister-like aneurysms with special reference to the flow-diverting strategy. Neuroradiology 2013; 55: 441-447. 
14) Chalouhi N, Zanaty M, Tjoumakaris S, et al: Treatment of blister-like aneurysms with the pipeline embolization device. Neurosurgery 2014; 74: 527-532; discussion 532.

15) $\mathrm{Hu} \mathrm{YC}$, Chugh $\mathrm{C}$, Mehta $\mathrm{H}$, et al: Early angiographic occlusion of ruptured blister aneurysms of the internal carotid artery using the pipeline embolization device as a primary treatment option. J Neurointerv Surg 2014; 6: 740-743.

16) Fiorella D, Albuquerque FC, Deshmukh VR, et al: Endovascular reconstruction with the neuroform stent as monotherapy for the treatment of uncoilable intradural pseudoaneurysms. Neurosurgery 2006; 59: 291-300; discussion 291-300.

17) Gaughen JR, Hassan D, Dumont AS, et al: The efficacy of endovascular stenting in the treatment of supraclinoid internal carotid artery blister aneurysms using a stent-in-stent technique. AJNR Am J Neuroradiol 2010; 31: 1132-1138.

18) Meckel S, Singh TP, Undrén P, et al: Endovascular treatment using predominantly stent-assisted coil embolization and antiplatelet and anticoagulation management of ruptured blood blister-like aneurysms. AJNR Am J Neuroradiol 2011; 32: 764-771.

19) Walsh KM, Moskowitz SI, Hui FK, et al: Multiple overlapping stents as monotherapy in the treatment of 'blister' pseudoaneurysms arising from the supraclinoid internal carotid artery: a single institution series and review of the literature. J Neurointerv Surg 2014; 6: 184-194.

20) Wang C, Tian Z, Liu J, et al: Flow diverter effect of LVIS stent on cerebral aneurysm hemodynamics: a comparison with enterprise stents and the pipeline device. $J$ Transl Med 2016; 14: 199. 